sam, irmanados, peritos criminalísticos, médicos legistas, policiólogos, juristas teóricos e práticos, professores, estudiosos e, enfim, todos quantos direta ou indiretamente estejam vinculados ao assunto, examinar e discutir com as necessárias elevação, liberdade, equanimidade e proficiência, os problemas, o presente e o futuro da Criminalística.

Não olvidemos, enfim, que o comodismo e o conformismo, de consórcio com o lugar comum, a ignorância e o preconceito, são a floresta virgem que, desbravada, ao menor discuido do desbravador avança e recupera com juros o que lhe fora subtraido, sepultando a obra pioneira na folhagem densa, nas lianas e no estrumes do seu desenfreado metabolismo. Não esqueçamos que nem mesmo cidades representativas de portentosas civilizações, como a legendária Tróia e a enigmática Machu-Pichu, puderam escapar a este destino. Por isso, saibamos responder ao desafio que, ora, nos é lançado, fazendo com que se desenvolva, em órbita mais ampla e transcendente, aquilo que em sentido restrito, se vem realizando, com respeito à Criminalística, nas Escolas de Polícia.

Recordemos, portanto, as cristalinas lições do emérito Bischoff (21), segundo o qual "A evolução da Polícia científica pode resumir-se da seguinte maneira: três períodos principais, dos quais o primeiro é o da identificação ods reincidentes; o segundo o período de aplicação de toda classe de procedimentos técnicos às investigações judiciais; $e$, por último, o terceiro período, que é o da extensão dos procedimentos verdadeiramente científicos, período de sistematização, de racionalização e, sobretudo, de ensino.": Não esqueçamos a lapidar advertência do renomado mestre, de que "uma disciplina que não é ensinada cessa de ser vivente, não tarde em deslocar-se, em ser presa do oportunismo e, por último, em perder-se na rotina, em lugar de viver e progredir".

Eis, pois, digamos, nas palavras do insigne De Rechter: "Metamos mãos à obra! Prossigamos, unindo todos os nossos esforços contribuindo ao avanço da criminologia, e para o seu seio conduzindo, também, material sólido e bem equilibrado, para ajudar à edificação de uma humanidade mais perfeita, mais desenvolvida no sentido da beleza, da verdade e da justiça".

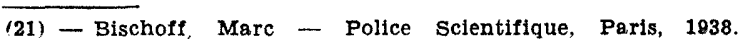

\title{
EXTRATOS DE UMA TESE *
}

\section{Prof. Plauto Faraco de Azevedo}

\section{L'ECOLE ANGLO-AMERICAINE}

1. Le développement tardif du droit international privé anglais et ses causes.

On appelle Ecole Anglo-Américaine le courant d'idées qut se forma en Angleterre et aux Etats Unis sous l'influence de I'Ecole Hollandaise des Statuts et surtout de la pensée de Huber. Quand on songe à ce courant de pensée du conflit de lois, un fait surtout frappe l'esprit - la formation lente, voire tardive de cette branche juridique en Angleterre. En effet, ce fut seulement après le XVIIe siècle que la loi étrangère fut admise, cà et là, de manière voilée, par les tribunaux anglais. ${ }^{1}$ C'est ainsi que l'on constate un décalage frappant entre la formation du droit international privé anglais et le développement de cette matière dans d'autres Etats de l'Europe continentale. En fait, cette branche de la loi anglaise n'a pris naissance qu'au milieu du XVIIIe siècle. ${ }^{2}$ Le fait étant constaté, rien de plus naturel que de se demander quelles en furent les causes. Pour ce faire il faut esquisser, d'une part, les raisons historiques, et d'autre part les motifs d'ordre strictement juridique.

Ainsi, l'on constate qu'il manqua en Angleterre la circonstance génératrice des conflits de lois en France et en Italie du Moyen-Age, c'est-à-dire l'existence de conflits interterritoriaux. Cela s'explique par l'établissement, à la suite de la conquête normande (1066), d'un gouvernement fortement centralisé, de telle manière que les tribunaux

(*) Reprodução de trechos da TESE "Recherches sur la justification de application du droit étranger chez les anglo-américains et leurs antécédents 28-01-1971, para a obtenção do título e grau de Doutor em Direito (publicada pelo "Centre de Drot lno do

3. A N. Sack Conflicts of Law in the History of the English Law, dans Law A N. Sack Conflicts of Law in the History of
Century of Progress 1835-1935, 1937, vol. 3, p. 398 . 
royaux s'arrogèrent une suprématie complète sur les tribunaux locaux. Par cette voie, les tribunaux royaux, ayant juridiction sur tout le territoire du royaume, finirent par établir une loi commune, "a common law", à tout le royaume, ce qui fut définitivement acquis au XII siècle.

Pour ce qui concerne les conflits internationaux, jusqu'au XVIIe siècle la règle établie fut que "de tels cas n'étaient pas susceptibles d'être jugés dans le domaine de la "common law". ${ }^{2}$ Cela s'explique par les traits caractéristiques du système judiciaire en vigueur autrefois en Angleterre. L'administration de la justice soit en matière civile, soit en matière pénale, se réalisait selon le "jury system". Ce système reposait sur une ancienne constitution du pays et consistait dans le jugement "per pais, c'est-à-dire, par le pays ("coun. try"), ce qui signifiait (dans l'ancien langage de la loi) lt comté ("county")". D'après lui un citoyen ne pouvait être atteint dans sa proprieté, sa liberté ou sa personne que par le consensus unanime de douze de ses voisins et égaux ("equals"). Ces juges, recrutés dans le voisinage, étaient des homens ayant une connaissance personnelle de la matière litigieuse. ${ }^{3}$ Le "jury system" empêchait la connaissance des actions fondées sur des faits accomplis à l'étranger, car les juges ne pouvaient évidemment être censés connaître les faits de la cause dans ces hypothèses. Cela provoqua l'observation de Nussbaum que le problème des conflits de lois $\mathrm{y}$ était, au début, de nature juridictionnelle. ${ }^{4}$

Ce système resta adapté aux conditions sociales de l'Angleterre jusqu'au XVIIe siècle. Mais, vers 1605, le régime social et économique fondé sur la propriété de la terre, l'agriculture et d'autres occupations à caractère local avait changé. La richesse de la nation était représentée, dans une large mesure, par différentes sortes de propriétés personnelles, situées non seulement en Angleterre mais aussi au-delà de la mer, à telle enseigne que la modification de l'ancien système s'imposait. ${ }^{5}$ A cause de ce besoin, les règles de jugement de la "common law" subirent des modifications graduelles. Le "jury" commenca à trancher les affaires non seulement selon la connaissance personnelle qu'en avaient ses membres, mais aussi en aceptant la déposition de témoins. En 1562 un "statute" établit le de-

1. A. N. Sack, Conflicts of Laws in the History of the English Law, dans Law a 2. "Such cases were not triable at the "common law". A. N. Sack, op. cit., p. 344 . 3. A. N. Sack, op. cit., p. 345 , note 40 et p. 346 .

4. A. Nussbaum, op. cit., p. $\mathbf{p}$. 1 voir du témoin de comparaître devant le juge quand cela fut jugé nécessaire et, à la fin du XVe siècle, le procès orienté la manière principale d'informapar obstacle à la connaissance des actions tion judiciaire. ${ }^{6}$ Cet obstacle à la connaissance des

Toutefois, il en restait un autre. Jusqu'au début du XVIIe siècle, époque où pour la première fois les tribunaux anglais commencèrent à faire face au problème des conflits de lois, prévalut l'dée selon laquelle les litiges ("causes") de lois, prévalut une loi déterminée devraient être jugés unigouvent par les tribunaux capables d'appliquer la loi en quement par les tribune "les tribunaux de la "commonquestion. Pácidaient que les litiges anglais, et leur appliquaient law" "ne decidaient " Ils ne décidaient pas d'autres litiges la "common law' d'autre loi que la "common law""

ni ne connaissaient d'autre lor que la "comm on avait trouvé

Relativement aux litiges commerciaux, on avait trouvé tranchés selon la "law merchant", consideree comme une lo commune à toutes les nations. Et cette loi, quoique considérée comme universelle [Malynes, Consuetudo vel Lex
Mercatoria or the Ancient Law Merchant (1622, 3 ed., 1686) ] était techniquement considérée comme étant de la loi anglaise. ${ }^{2}$ Dans ce sens il est intéressant de remarquer qu'au début, la "Lex Mercatoria" était appliquée seulement par les tribunaux de "l'Admiralty" et par des tribunaux de commerce spéciaux. Cependant, à partir de 1660 ("Restoration"), les tribunaux de la "common law" avaient prestoration"), les tribur cas fondés sur la "law que exclusivement jurivéquence en fut la suivante: les régles de cette loi cessèrent graduellement d'être considérées comme des questions de fait jusqu'à devenir "une partie de la "common law"" Ainsi, le principe resta intact: "les tribunaux de la "common law" n'appliquent encore que leur propre loi, la "common law"!". La "Law Merchant" fut recue, incorporée dans la "commor law". ${ }^{3}$

Même sans vouloir entrer dans les détails, ce que les limites de la présente recherche ne permettraient pas, il faut relever le fait qu'encore au XVIIe siècle, la situation était confuse pour ce qui concerne la solution des cas présentant des éléments étrangers à l'Angleterre. Fréquemment, il était défendu aux cours de "l'Admiralty" de trancher ce

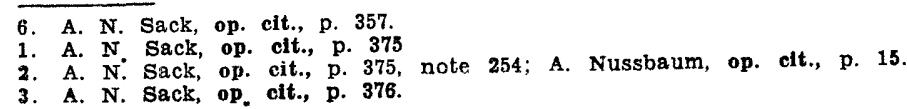


genre de cas; d'autre part, les tribunaux de la "common law" hésitaient à en assumer la juridiction. ${ }^{4}$ Et les cas ayant des éléments étrangers ne faisaient que s'accroître. La réalité n'attendait donc pas la lente évolution des idées juridiques. Elle allait de l'avant et demandait résolument sa reconnaissance. Malgré sa demande pressante, la réponse juridique ne fut que partielle. La doctrine, graduellement, établit que les tribunaux anglais devraient, dans certaines hypothèses, donner effet en Angleterre aux jugements étrangers, à condition que ceux-ci fussent rendus par les tribunaux étrangers ayant compétence pour juger ces cas c'est-à-dire capables d'appliquer la loi étrangère en question. ${ }^{1}$ En d'autres mots, les jugements concernant les litiges ayant des éléments étrangers devraient être rendus par les tribunaux étrangers compétents et, par la suite, on reconnaissait la force obligatoire de ces jugements en Angleterre.

La réflexion théorique, dans le champ du droit international privé, ne commença que dans la deuxième partie du XVIIIe siècle, à travers quelques jugements de Lord Mansfield, fondés surtout sur la pensée de Jean Voet et de $\mathrm{Hu}$ ber. ${ }^{2}$ Mais l'élaboration de ce qui ressemblerait à un système cohérent de conflits de lois fut lente et laborieuse. ${ }^{3}$

Il faut relever encore la circonstance que le premier ouvrage sur la matière ne parut qu'en 1823: Jarbez Henry, "The Judgement of the Court of Demerara in the Case of Odwin v. Forbes, to which is prefixed a Treatise on the Difference Between Personal and Real Statutes, and its Effect on Foreign Judgements and Contracts, and Marriages and Wills"; William Burge ne publia son ouvrage qu'en 1838: "Commentaries on Colonial and Foreign Laws Generally, and in Their Conflict with Each Other and with the Laws of England", 4 vol. Ce n'est qu'au milieu du XIXe siècle, en 1858, que Westlake publia son ouvrage intitulé "Private International Law, with Principal Reference to Its Practice in England". Les "Commentaries upon International Law", de Sir Robert Philimore, dont le quatrième volume se rapporte aux conflits de lois et s'intitule "Private International Law Or Comity", apparurent en 1861. Par contre aux Etats Unis, en 1828, Samuel Livermore avait déjà publié son ouvrage intitulé "Dissertation on Questions which

\section{A. N. Sack, op. eit., p. 381.
A. N. Sack, op. cit., p. $381-382$.}

G. C. Cheshire, Private International Law. 7e Ed, London, 1965, p. 36.
Arise from the Contrariety of the Positive Laws of Different States and Nations"; et, en 1834, Story publia son ouvrage devenu celèbre et intitulé "Commentaries on the Conflict of Laws. Foreign and Domestic"."

2. Le développement tardif du droit international privé anglais et la jurisprudence.

L'évolution tardive du droit international privé anglais vient d'être esquissée dans ses grandes lignes. Il est maintenant loisible de montrer rapidement les difficultés auxquelles s'est heurtée la jurisprudence en raison de présupposés théoriques décrits ci-dessus. En voici quelques arrêts.

Ainsi, encore en 1625, dans Ward's case, 1 le demandeur fit état d'une dette fondée sur une facture dressée à Saint Mary Le Bow à Londres dont il réclamait le paiement. En fait, la facture avait été faite à Hambourg. Le jugement fut favorable au demandeur, quoique en utilisant un artifice. Le juge déclara que pour pouvoir trancher ce cas, ou autrement dit, pour assurer la jurisdiction de son tribunal sur ce cas il fallait considérer que Hambourg se trouvait à Londres. Ainsi, il dit ceci: "bien que nous sachions que Hambourg se trouve au-delà de la mer, comme juges, nous ne prenons pas en considération que cette ville se trouve au-delà de la mer" ?

Dans Foubert v. Turst, ${ }^{3}$ tranché en 1703 , le litige portait sur le régime matrimonial d'un ménage francais, établi par contrat, en 1665, en France. Ce contrat stipulait la communauté de biens à partir du mariage, selon la Coutume de Paris. En outre, une dot d'um montant de 1.200 livres sterlings fut donnée au mari; un tiers devait être intégré à la communauté de biens et les deux tiers restant constituaient le patrimoine personel de l'épouse. Comme aucun enfant ne naquit de cette union, les conjoints se mirent d'accord pour changer le régime de biens auparavant convenu, dressant, à cet effet, em 1680 , un autre contrat d'après lequel, afin que "le conjoint survivant puisse avoir la plus complète subsistance, il était convenu que celui-ci recevrait et jouirait de tous les biens et droits qui leur étaient communs, en accord avec la Coutume de Paris, à condition qu'il n'y ait pas d'enfants issus de cette union"."

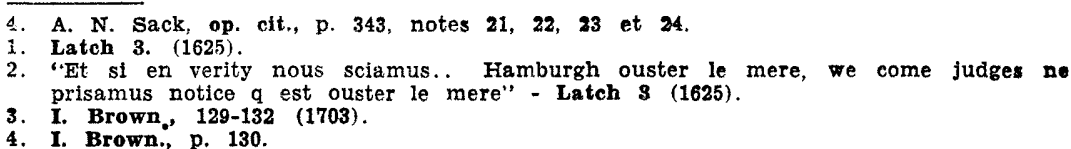


Après le mariage, les conjoints s'établirent en Angleterre comme réfugiés et dans ce pays la femme mourut sans avoir d'enfant. Ses parents assignèrent le mari en paiement de 800 livres sterlings, à titre de propre de l'épouse selon les termes de la dot, et la moitié des biens du ménage car ils estimaient que le deuxième contrat ci-dessus mentionné n'était pas valable, étant donné que le mari n'était pas retourné "dans le délai légal à Paris pour y réaliser un inventaire de ses biens". ${ }^{1}$ Le mari défendeur allégua pour sa part que le premier contrat était nul et qu'en accord avec le deuxième, il avait droit á tous les biens communs; il prétendait, au surplus, qu'étant um réfugié on ne pouvait pas Iui appliquer la Coutume de Paris par laquelle il était frustré, mais la loi anglaise selon laquelle il avait droit à tous les biens.

Lord Keeper Wright soutint le point de vue du mari. Cependant, les parents de l'épouse, sur appel, insistèrent sur leur prétendu droit et la "Court of Chancery" condamna le mari à leur payer les 800 livres sterlings correspondant à la partie propre de l'épouse de même que la moitié des biens du ménage.

Malgré l'opinion divergente das parties concernant l'objet du litige, toujours est-il que les arguments de l'une et l'autre envisagèrent le problème de l'application de la loi étrangère de la même façon. En effet, le mari défendeur déclara "qu'il serait étrange... qu'un tribunal fut obligé d'appliquer de telles lois et coutumes françaises";: les parents demandeurs, à leur tour, affirmèrent que leur but était l'exécution du contrat de mariage et que cela ne signifiait "en aucune manière une tentative d'introduire des lois ou des coutumes étrangères comme ayant vigueur en Angleterre". ${ }^{3}$ Ainsi, comme l'observe justemente Sack, le cas fut traité comme ne se rapportant qu'à l'exécution d'une prévision d'un contrat sans que l'on y songeât à la possibilité d'appliquer une loi étrangère. ${ }^{4}$

Comme souligne Nussbaum, ce ne fut que plus tard que l'on put se fonder sur la loi étrangère elle-même, mais son approche comme fait ne fut jamais abandonnée. D'après cet auteur,cette manière de concevoir le problème de l'applition de la loi étrangère fut déjà suivie dans Dunganon v.

Hackett tranché en 1702. Il y fut décidé que l'intérêt corres-

$\begin{array}{lll} & \\ \text { 1. } & \text { I. Brown., p. } 130 . \\ \text { 2. } & \text { I. Brown., p. } 131 . \\ 3 . & \text { I } & \text { Brown, p. }\end{array}$

4. A. N. Sack, op. eit., p. 388 pondant à une dette contractée en Irlande devait être payé selon la loi de ce pays. Apparemment, la loi irlandaise fut considérée comme une des circonstances de fait ayant une influence sur le taux de l'intérêt. ${ }^{5}$

Comme il est impossible de considérer tous les cas dans les limites de cette recherche, il parait préférable d'attirer l'attention sur quelques-uns de ceux qui furent tranchés durant cette époque de formation du droit international privé en Angleterre.

Dans Robinson v. Bland, tranché en $1760,^{1}$ un citoyen britannique avait prêté à un autre la somme de 300 livres sterlings, "au moment et au lieu du jeu de hasard". Cette somme fut ensuite perdue au jeu, au même endroit, au profit de celui qui l'avait prêtée, avec un surplus de 372 livres sterlings. Une lettre de change couvrant toute la somme, soit 672 livres sterlings, fut alors émise à Paris pour être payée en Angleterre. Après la mort de son souscripteur, une action fut intentée en Angleterre contre "l'administrateur" ("the administrator") se fondant sur la lettre de change ainsi que sur le prêt d'argent. La décision en fut que la lettre de change était nulle et qu'il n'existait pas d'action assurant la possibilité de récupérer l'argent gagné au jeu. Elle donna cependant gain de cause au demandeur en ce qui concerne le prêt. Comme les trois juges qui connurent de l'affaire se prononcèrent séparément, il convient de considérer individuellement chacune de leurs opinions, en prêtant une attention spéciale à celle de Lord Mansfield. Celui-ci commence par dire que le cas ne pose pas de problème particulièrement difficile car les lois anglaises et françaises donnent la même solution au litige. Ensuite, il divise l'affaire en deux parties, à savoir la considération de la lettre de change et puis la question de savoir si le demandeur avait droit à récupérer quelque chose "en vertu de la justice et de l'équité de son cas", indépendamment de la lettre de change. En ce qui concerne celle-ci "les parties avaient en vue les lois d'Angleterre" et "la loi du lieu ou l'acte a été passé ne peut jamais régler le cas quand la transaction fut réalisée avec mention expresse à la loi d'un autre Etat comme étant celle par laquelle elle doit être régie". ${ }^{2}$ Lord Mansfield s'appuie ici sur des textes de Huber, en faisant la citation. De plus, "la règle générale, établie ex comitate et jure gentium, 5. A. Nussbaum, op. eit., p. 16.
1. 2 Burr., $1077-1088$ (1760). 
c'est que l'on doit considérer plutôt le lieu où le contrat est passé que celui où l'action est intentée, afin d'interpréter et sanctionner le contrat. Cependant, cette règle admet une exception quand, au moment de conclure le contrat, les parties avaient en vue un autre royaume". ${ }^{3}$ Ainsi, la loi d'Angleterre ayant été choisie et la lettre de change étant nulle conformément à celle-ci, le demandeur ne saurait rien demander sur base de cet effet de commerce.

Pour ce qui concerne le deuxième aspect ci-dessus mentionné, Lord Mansfield considéra que le demandeur ne saurait pas réclamer devant un tribunal anglais l'argent gagné au jeu de hasard; d' après la lol française, le contrat vu sous cet aspect était également nul, la question de savoir laquelle des deux lois en question devrait être appliquée pour résoudre le litige était oiseuse. Néanmoins, la justice et l'équité du cas pouvaient être considerées sous un autre jour, à savoir le prêt d'argent. Et sa conclusion fut que si le demandeur n'avait aucun droit sur l'argent gagné au jeu, par contre le prêt d'argent l'autorisait à réclamer la somme correspondante. Sur ces deux points les lois en question - française et anglaise - se trouvaient en accord.

Néanmoins, on se rend bien compte que l'identité de deux lois avec lesquelles le cas gardait des liens, n'a absolument pas empêché Lord Mansfield de l'envisager dans une optique moderne, tout en considérant de façon très logique chacun de ses aspects. En effet, il distingua nettement les problèmes de la compétence juridictionnelle et de l'application de la loi compétente, ce qui, à l'époque, n'était pas facile, à telle enseigne que les deux autres juges qui connurent de l'affaire ne les đistinguèrent pas de façon satisfaisante. De fait, les juges Denison et Wilmot étaient d'accord que le demandeur ne saurait fonder son action que sur le prêt Cependant, leur accord s'étendit au problème de la loi applicable car ces deux juges estimèrent que, comme le demandeur avait intenté le procès en Angleterre, cela impliquait l'application de la loi anglaise pour trancher l'affaire. Autrement dit, au choix du for anglais devait s'ensuivre la supposition du choix de l'application de la loi anglaise pour trancher le différend.

Cheshire observe que l'opinion exprimée par Lord Mansfield fut la première prise de position en faveur de la loi de l'autonomie pour régler le contrat. En revanche, il est à

3. I Wm. Bl. 258-259, dans G. C. Cheshtre, op. elt., p. 36. noter que jusqu'en 1760, "les règles sur un problème tellement important n'étaient pas du tout établies". 1

En 1774, dans Mostyn v. Fabricas, ${ }^{2}$ il fut établi qu'une action, fondée sur l'acte illicite de détention arbitraire ("trespass and false imprisonment") commis par le gouverneur de l'ile de Minorque contre un de ses ressortissants, pouvait être intentée en Angleterre. Dans ce cas le demandeur se plaignit d'avoir été attaquẻ par le défendeur, mandeur se plaignit la force et les armes, l'avait frappé, maltraité et blessé, à Minorque, "c'est-à-dire à Londres, dans la paroisse de St. Mary le Bow, dans le quartier de Cheap", ${ }^{3}$ et parois l'avait enfermé en prison pour une période de dix après, l'avait enferme en prison pour une pén mois sans en avoir aucun motif raisonnable et enfin, l'avait banni de Minorque, lieu de sa résidence et demeure habituelle, l'envoyant à Cartagena. Le défendeur allégua en revanche, qu'il était à l'époque le gouverneur de Minorque et que c'est à ce titre qu'il avait agi car le demandeur étalt coupable de sédition: il avait essayer d'ameuter la populacion de Minorque. Et que, pour préserver la paix, en tant que gouvorque. Et que, il avait ordonné que le demandeur fût banni de l'ile; pour ce faire, il avait ordonné, auparavant, l'arrestation du demandeur pour une courte période après laquelle on l'avait mis à bord d'un vaisseau et envoyé à Cartagena. Le défendeur prétendait que tous ces actes avaient été accomplis en accord avec la loi et dans les limites de son pouvouir de gouverneur de Minorque.

Le problème le plus important soulevé par ce litige était de savoir si l'on pourrait intenter un procès en An gleterre en raison d'une arrestation ayant eu lieu à Minorque, contre un ressortissant de cette île.

Pour résoudre le problème, l'on examinera une fois de plus l'opinion de Lord Mansfield qui apporta à la formation théorique du droit international privé anglais une contribution décisive.

Il commence par remarquer que s'agissant d'un cas dont les éléments étaient disséminés à travers plus d'un Etat vu que l'agression et la détention arbitraire à Minorque avaient été suivis du bannissement à Cartagena, il était nécessaire au demandeur de déterminer "le vrai lieu où le motif de l'action est né: par conséquent, il afirma qu'il était à Minorque; avec un c'est-à-dire, à Londres, dans la paroisse 1. C G. Cheshire, op. cit., 7e. ed., London, 1965, p. 37 3. "... at Minurca, (to wit) at London aforesald, in the parish of st. Mary le ... 
de St. Mary le Bow, dans le quartier de Cheap". ${ }^{1}$ Ensuite, Lord Mansfield considéra les allégations du défendeur, dont il faut en relever deux: a) le défendeur estimait qu'en sa qualité de gouverneur de Minorque, il ne pouvait être jugé responsable d'aucun acte illicite commis par lui dans l'exercice de ses fonction; b) que l'acte illicite ("injury") ayant été commis à Minorque, en dehors du royaume, il n'était pas susceptible d'être connu par les tribunaux royaux en Angleterre. Sur ce, Lord Mansfield fait des considérations remarquables, qui démontrent une extrême logique de raisonnement de même qu'un sens aigu de la justice et une sensibilité spéciale pour résoudre des cas présentant des éléments étrangers. C'est ainsi qu'il argumente que, pour refuser d'accorder la juridiction des tribunaux anglais sur un cas quelconque, il était nécessaire de démontrer l'existence d'une juridiction plus appropriée et, en revanche, s'il n'y avait pas une autre façon de trancher le litige, cela suffisait à donner juridiction aux tribunaux anglais, ce qui était bien le cas de l'affaire pendante devant le tribunal. En outre, i estimait que si les actes passés à l'intérieur d'un dominion devaient être jugés dans ce dominion "l'effet et l'extension des letres patentes de Sa Majesté, lesquelles accordaient le pouvoir (au gouverneur), ne pouvaient être jugés que par les tribunaux de sa Majesté...".2 De cette facon Mansfield soutint le point de vue du demandeur, lui accordant la juridiction anglaise, en dépit de ce que l'acte illicite s'était passé à Minorque. Ensuite, réfutant la première allégation du défendeur, ci-dessus exprimée, Lord Mansfield estima qu'il devait prouver, d'après le droit de Minorque, qu'il n'avait commis aucun acte illicite. Il établit alors cette règle depuis lors suivie, d'après laquelle "tout ce qui constitue une justification dans l'endroit où le fait s'est passé devrait être une justification où l'action est intentée" 1 De tels raisonements permirent au droit international privé anglais d'aller de l'avant, laissant dans son sillage de vieilles conceptions qui ne satisfaisaient plus aux nouvelles exigences sociales dont l'accroissement des rapports avec le continent n'était qu'un symptôme. C'était, au surplus, laisser de côté des artifices surannés comme on en trouve un échantillon dans la détermination du lieu de l'acte illicite par le de-

1. " $\ldots$ the real place where the cause of action arose: therefore, he has stated
it to be in M.inorca; with a videlicet, at London, in the parish of st. Mary le
Bown, in the ward of Cheap". - Idem, p. 170. Bown, in the
Idem, p. 173. "... whatever is a justification in the place where the thing is done ought to be a mandeur à Minorque, mais avec un "c'est-à-dire" ("videlicet"), à Londres, à Saint Mary le Bow. Bref, c'était laisser de côté les arguments purement formels pour s'attaquer de façon lucide aux problèmes posés par un monde qui changeait et demandait des solutions juridiques en rapport avec ce changement.

Dans Scrimshire ${ }^{2}$ v. Scrimshire qui date de 1752 , le for anglais examina très soigneusement le cas d'un mariage clandestin célébré en France entre deux sujets britanniques, sur base d'une demande de restitution des droits conjugaux effectuée par la femme, celle-ci argumentant que le mariage avait été célébré à l'étranger sans l'accomplissement des lois du lieu où l'acte s'était passé. Les lois des deux Etats le tenaient pour nul. Selon la loi francaise, la nullité était absolue, tandis que la loi anglaise était plus nuancée sur ce point. Le Parlement de Paris avait, d'ailleurs, déjà déclaré la nullité du mariage.

Sir Edward Simpson examina méthodiquement chacun des aspects du litige, observant dès le début qu'il s'agissait d'un cas dont l'intérêt dépassait les parties pour retentir dans le domaine de l'intérêt public. Il commence par considérer que, si le but du procès était la restitution des droits conjugaux, il posait une question préliminaire, à savoir l'existence du mariage en vertu duquel les droits conjugaux étaient réclamés. Ainsi, il estima que la considération des lois françaises était très pertinente pour déterminer, selon la loi anglaise, la validité du contrat de mariage réalisé en France, ${ }^{3}$ et que celui-ci était vraiment clandestin car il avait été célébré entre des mineurs, sans le consentement des parents, dans une maison particulière, par un prêtre qui n'avait pas l'autorisation légale nécessaire, ce qui n'était admis par aucune des deux lois en question. Cependant, celles-ci contenaient une différence à cet égard. Selon la loi anglaise, le mariage, tel qu'il avait été célébré en France, aurait pu être considéré valable (bien que les parties et le prêtre étaient susceptibles de punition) si la loi francaise et le Parlement de Paris ne le considéraient nul. C'était donc sur ce point, que se trouvait la difficulté de valider le mariage en Angleterre.

Un autre aspect analysé par Sir Edward Simpson était l'allégation d' après laquelle les deux parties étant de na-

2. 2 Hag. Con. $395-422$ (1752). "ass I think the laws of France are very material to be considered, in determining,
even by our law, on the validity of a contract of marriage had and made in
France". - Idem, pp. 398 . 
tionalité britannique et non domiciliées en France, le seul motif qui pouvait les assujétir à l'obéissance aux lois françaises s'évanouissait. A quoi le juge répondit que le problème ne concernait pas, ni ne dépendait de la détermination du domicile des parties, comme la question n'était pas de savoir si les citoyens britanniques devaient obéir aux lois trançaises, "car, sans aucun doute, aucune loi française ne peut astreindre des citoyens anglais qui ne se trouvent pas sous son empire". 1 Tout en écartant cet argument, le juge résuma le problème devant le tribunal comme n'étant que celui de savoir si le mariage en question était valide ou non selon les lois d'Angleterre. ${ }^{2}$ Pour résoudre cette question, le juge affirma que la loi anglaise stipulait que la loi étrangère devait être prise en considération dans des cas pareils De cette manière, la considération de la "lex loci celebrationis" pour déterminer la validité formelle du mariage ne signifie pas que les droits des citoyens anglais sont déterminés par la loi française, mais par la loi anglaise qui sanctionne et adopte cette règle de décision. ${ }^{3}$

Néanmoins, si la "lex loci celebrationis" fut considérée comme la loi appropriée à déterminer la validité formelle du contrat de mariage, elle n'était quand même pas appliquée par elle-même, mais "sanctionnée et adoptée par la loi du for comme sa règle de décision".4

La règle établie, il falait encore motiver la considération de la loi étrangère qu'elle entraînait dans le cas d'espèce, ne fût-ce que pour façonner la règle de décision du for Dans ce sens, le juge anglais se demande: "pourquoi ce tribunal ne peut-il pas prendre connaissance de lois étrangères, s'il n'y a rien d'illégal dans ce procédé?". ${ }^{5}$ Néanmoins, ce qui frappe l'attention est la voie qui fut choisie par ce magistrat pour justifier cette proposition. Il y argumente que la considération de la "lex loci celebrationis" dérive du consentement de tous les Etats, donc relève du jus gentium or, comme celui-ci fait partie de la loi anglaise, en suivant la règle qu'il prescrit, on ne fait autre chose qu'appliquer exclusivement la loi anglaise. ${ }^{6}$

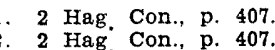

. "If such be the law of this country, the rights of English subjects cannot be said to be determined by the laws of France, but by those of their own country, whic sanction and adopt this rule of decision". - 1em, p. 408.

4. "2 Hag. Con,, p. 408 Court then take notice of foreign laws, there being nothin illegal in doing it?" - Idem, p. 416 .

6. 2 Hag, Con., p. 416
}

Dans Holman v. Johnson, ${ }^{1}$ en 1775 , les demandeurs, ayant vendu et livré au défendeur une portion déterminée de thé à Dunkerque tout en n'ignorant pas que celui-ci avait l'intention de faire la contrebanaie de la marchandise en Angleterre, saisirent le for anglais pour recouvrer le prix de vente du thé. Pour compléter le cadre de fait du cas en question, jl faut ajouter que si les demandeurs connassaient les intentions du défendeur, ils se bornèrent à accomplir un acte de commerce normal sans se mêler de la contrebande.

C'est encore Lord Mansfield qui saisit l'occasion de formuler de façon non équivoque la doctrine moderne du droit international privé anglais. En effet, il commença par affirmer: "il n'y a aucun doute que tous les litiges tranchés ici doivent l'être en accord avec la loi anglaise; mais la loi anglaise dit que, dans plusieurs circonstances, pour ce qui concerne les contrats régulièrement passés à l'étranger, les lois de l'Etat où la cause de l'action surgit doivent les régir". ${ }^{2}$ Néanmois, s'il posa de façon si nette cette règle sans aucun doute très importante pour le développement du droit international privé anglais et s'll affirma qu'il s'agissait d'un principe admis par tous les Etats, il l'écarta pour ce qui concerne la solution du litige pendant devant le tribunal. Pour le trancher il estima devoir examiner la transaction effectuée à Dunkerque, ci-dessus mentionée. Le contrat fut dûment exécuté, par la livraison de la quantité de thé promise, et l'action ne fut intentée que pour recouvrer le prix de la marchandise ainsi vendue à Dunkerque. Les vendeurs savaient ce que l'acheteur allait en faire, mais ils ne se sont livrés à aucune action dans ce sens. Bref, l'intérêt des vendeurs s'était épuisé dans la vente et le contrat s'était achevé par la livraison des marchandises à Dunkerque. De cette facon, les demandeurs n'avaient offensé aucune disposition légale anglaise. Argumentant dans ce sens, Lord Mansfield fait la citation du texte de Huber dans lequel le juriste hollandais affirmait (en conformité avec son principe général de validité ou nullité universelle des actes juridiques valides ou nuls "ab initio" selon la loi où ils ont été accomplis) (p. 28, note 1) que, si dans un Etat déterminé, la vente d'une marchandise est inderdite, la vente réalisée dans cet Etat est nulle et, en revanche, si les mêmes marchandises

1. I Cowp. 341-345 (1755).

but that every action tried here must be tried by the law of England; but the law of England says, that in a variety of circumstances,
with regard to contract legally made abroad, the law of the country where the with regard to contract legally made abroad, the law
cause of action arose shall govern" - Idem, p. 343 . 
sont vendues dans un autre Etat où la transaction est permise, la vente est valable et l'acheteur en est responsable. Après quoi, Lord Mansfield introduit la pensée de Huber dans le cas "sub judice" en la "traduisant" de la facon suivante: "En Angleterre, le thé qui n'a pas payé la taxe est prohibé et, s'il y est vendu, le contrat est nul. Cependant, s'il est vendu et livré dans un lieu où il n'y a pas d'interdiction, par exemple à Dunkerque, et si une action est intentée pour avoir son prix en Angleterre, l'acheteur doit être condamné à payer ce prix car le contrat qui en est à l'origine est bon et valide". 1

Sir William Scott (plus tard devenu Lord Stowell) dans le cas de Dalrymple v. Dalrymple, ${ }^{2}$ décida en 1811 qu'un mariage ayant eu lieu en Ecosse, par contrat, sans célébration religieuse et valable selon la loi de ce pays, était aussi valable en Angleterre. Il y formula sa fameuse affirmation que "étant admis dans un tribunal anglais, ce cas doit être tranché en accord avec les principes de la loi anglaise qui lui sont applicables. Cependant, selon la loi anglaise, le seul principe applicable à un tel litige est que la validité des droits matrimoniaux de Miss Gordon doit être jugée en tenant compte de la loi du pays où, s'ils existent effectivements, ils ont pris naissance. Ayant fourni ce principe, la loi anglaise s'éloigne tout à fait, laissant la question légale au jugement exclusif de la loi écossaise". 3 En fait, la même affirmation avait déjà été clairement faite par Lord Mansfield dans Holman v. Johnson.

Donc, on se rend facilement compte que le XVIIIe siècle représente la période embryonnaire du droit international privé anglais. Cette période va s'étendre au moins jusqu'au milieu du siècle suivant, quoique "la fin de la période de formation ne soit pas encore perceptible".4 Significative à cet égard est l'assertion faite en 1825 par C. J. Best dans Arnott v. Redfern" que "ces questions de droit international n'arrivent que rarement", 6

Huber, De Conflictu Legum, par. 5, dans D. J. L. Davies, op. eit., p. 68

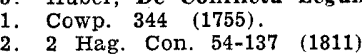

3. "Being entertained in an English Court,this case must be adjudicated according to the principles of English law applicable to such a case. But the only principle applicable to such a case by the law of England is, that the validity of Miss Gordon's

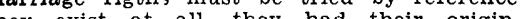
aw of England withdraws altogether and leaves the legal question to the exclusive judgment of the law of Scotland" - Idem, p. 58-50.

6. "these questions of international law do not often occur" - Idem, p. 90.
Le principe de l'application exclusive de la loi du for, ou, dans le langage employé par les auteurs anglo-saxons, l'application exclusive de la loi à laquelle doit obéissance le tribunal saisi, persiste en Angleterre encore de nos jours dans la matière du divorce. Les tribunaux anglais ne connaissent que les cas de divorce de personnes domicilées en Angleterre et ils n'appliqueront dans cette matière que la loi anglaise. La conséquence en est que pour ce qui concerne le divorce, l'occasion ne se présentera pas de considérer une loi étrangère.7

IV UNE VALORISATION DES THEORIES CONSIDEREES DANS UNE PERSPECTIVE HISTORIQUE

1. L'approche historique.

Considérant que l'exposé des théories envisagées fut fait selon une perspective historique, il reste à indiquer pourquoi une telle perspective fut choisie. De même, il est nécessaire de montrer à quelles constatations une telle approche est susceptible de mener.

\subsection{LE POURQUOI DE CETTE APPROCHE}

Depuis le début de la présente recherche, l'approche choisie fut justifiée par la croyance qu'il est impossible de compreendre n'importe quelle idée juridique si l'on ne la met pas en rapport avec la réalité historique dans laquelle elle vit le jour. En effet, parmi les buts de cette étude, le premier est de relancer la pensée d'après laquelle les théories et les règles juridiques ne sauraient être vraiment comprises qu'à condition de les rapprocher du milieu où elles sont nées et dont elles reflètent les tendances et les intérêts de tous ordres ainsi que les idées qui en découlent, voire les préjugés créés par la force de la tradition. Toute théorie juridique, de même que les règles qui en résultent, ne fait que répondre à des besoins déterminés et propres à chaque phase historique. Certes, on pourrait se dire qu'il s'agit là d'une vue bien connue. Néanmoins, il est toujours opportun d'insister sur cet aspect car, si son évidence est incontestable, on peut toujours observer la tendance à l'oublier. Cette tendance s'exprime soit par l'idée que la règle de droit peut être expliquée par elle-même, soit par la pensée que n'importe quelle règle de droit une fois posée par le pouvoir 7. A. N. Sack, op. eit., p. $397-398$, note 386 
politique, se trouve de ce fait, justifiée. Rien de plus erroné et dangereux. La règle juridique ne peut être justifiée que par son adéquation à la situation sociale qu'elle doit régler. Evidemment, si par sa mise en oeuvre, son inadéquation à la situation sociale visée apparait, cela n'implique pas qu'on pourrait pour autant nier sa force obligatoire. Mais c'est alors que la tâche du juriste doit devenir combative. Il lui incombe de démontrer le caractère insuffisant ou injuste de la règle de droit et de proposer une autre réglementation en conformité avec la situation sociale en question.

Bien que les cas de pathologie juridique soient exclus des limites de la présente recherche, il faut tout de même les rappeler car là, le problème sur lequel il faut attirer l'attention est plus apparent et la responsabilité du juriste en est d'autant plus grande. De nos jours, on peut constater l'existence de systèmes juridiques presque tout entiers non adéquats aux circonstances sociales qu'ils visent à régler. Et l'on constate l'attitude des juristes maintes fois caractérisée par son caractère conformiste vis-à-vis de pareils systèmes, ce qui constitue une raison de plus pour affirmer que la nécessité du rapprochement entre droit et réalité historique s'impose.

La règle juridique exprime un jugement de valeur porté sur la situation sociale qu'elle doit régir. On peut donc dire qu'elle est le résultat d'un choix entre diverses évaluations. C'est sur les raisons orientant ce choix que doit porter le jugement du juriste car la loi ne sera bonne que si elles sont historiquement vraies. Par circonstances historiques, on doit entendre toutes les forces sociales existant à un moment déterminé, à savoir économiques, politiques, morales, sociologiques, scientifiques, religieuses et historiques strictu sensu. Chaque loi rendra sensible plutôt à l'une qu'à l'autre de ces données bien qu'elle puisse se répercuter sur plusieurs secteurs de la réalité sociale, tandis que la constitution les rassemble toutes à la fois.

Ces quelques considérations ont pour finalité de mettre l'accent sur la position selon laquelle droit et histoire sont inséparables. Autrement dit, le phénomène juridique vient à la lumière pour determiner le modus vivendi des individus dans des phases historiques diverses. De fait, de même que l'on ne peut pas songer à la vie en société sans qu'il y existe des règles juridiques, la règle de droit perd son sens quand on méconnait les circonstances historiques auxquelles elle répondit ou doit répondre.
Ce rapprochement fut, par ailleurs, déjà fait de beaucoup de manières. Coing. ${ }^{1}$ résumant les idées de Collingwood dit que, d'après ce dernier, "l'histoire est l'empire de la liberté humaine" et que "l'homme fait ses décisions toujours en vue d'une situation bien déterminée" mais "c'est l'image que l'homme se fait de la situation plutôt que la situation elle-même, qui restreint sa liberté et détermine son action". Or, il résulte que ces principes sont applicables à l'interprétation du droit actuel car le législateur ainsi que le juge visent des situations sociales déterminées. Mais ce qu'il importe d'en retenir, c'est l'image qu'ils se font de la situation car "il n'est pas du tout assuré que cette image corresponde exactement à la réalité". ${ }^{2}$ Ensuite, Coing énonce l'opinion que "l'interprétation historique de l'acte législatif est indispensable". " Pour la réaliser, que faut-il faire ? Il est nécessaire de reconstruire la pensée du législateur, ce qui signifie qu'il faut se demander quelles ont été les catégories qui déterminèrent l'image que le législateur s'est fait de la situation. Or ceci comprend notamment les théories sociales en vigueur à l'époque de l'élaboration de la loi, la tradition juridique, les moyens techniques employés, des idées morales en vigueur, ainsi que des intérêts, soit politiques, soit économiques, qui influencèrent la législation. De ce fait, "là tâche du juriste qui cherche à interpréter le droit en vigueur est, par essence, historique" ${ }_{4}$

Il importe de souligner que cette règle, dégagée par des auteurs qui ont eu en vue la mise en oeuvre du droit matériel, est également valable en ce qui concerne les règles de conflit. ${ }^{5}$

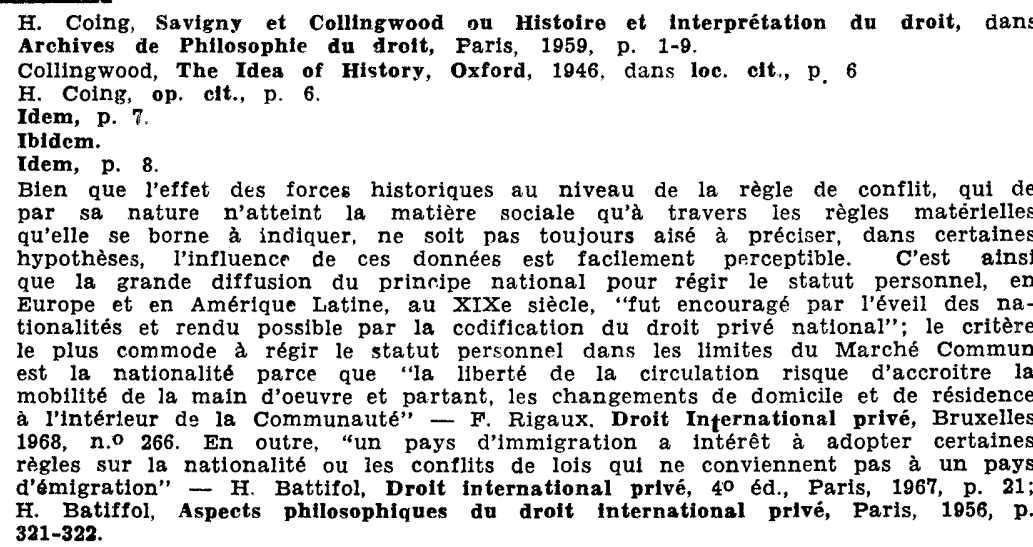

Bien que l'effet des forces historiques au niveau de la règle de conflit, qui de par sa nature n'atteint la matiere sociale qu a travers les règles matèrtelle qu'elle se borne a indiquer, ne sol pas touthes, l'influence de ces données est facilement perceptible. C'est ains que la grande diffusion du prinnipe national pour régir le statut personnel, en Europe et en Amerique Latine, au XIXe secle, "Iut encouragé par l'eveil des nadu Marché Commu est la nationalité parce que "la liberté de la circulation risque d'accroitre la mobilité de la main d'oeuvre et partant, les changements de domicile et de résidence à 1968 n.o 266. En outre, "un pays d'immigration a intérêt à adopter Bruxelles règles sur la nationalité ou les conflits de lois qul ne conviennent pas à un pays d'émigration" - H. Battifol, Droit international privé, 40 éd., Paris, 1967, p. 21 321-322. 


\subsubsection{L'Ecole Anglo-Américaine.}

Pour ce qui concerne l'Ecole Anglo-Américaine, on trouve des exemples vraiment saisissants de l'influence des circonstances historiques sur la formation des règles de droit international privé dans le système de la "common law". C'est ainsi qu'on put constater que le développement tardif du système de conflit de lois anglais fut dû, pour une grande partie, à l'inexistence des conflits interterritoriaux comme conséquence de la centralisation qui suivit la conquête normande ainsi qúà la difficulté, de la part des tribunaux anglais, de connaître des litiges concernant les conflits internationaux en vertu de l'existence de l'institution du "jury system", laquelle impliquait une connaissance personnelle de la matière "sub judice". En outre, on peut se rendre compte que ce ne fut que quand les circonstances socio-économiques se modifièrent, au XVIIe siècle, que les litiges concernant les relations privées internationales commencèrent à être connus, bien que la réflexion théorique ne commença que dans la seconde moitié du XVIIIe siècle à travers quelques jugements de Lord Mansfield. La jurisprudence donne même des exemples d'un effort très grand pour concilier l'idée de justice avec la tradition historicojuridique anglaise. En fait, on ne pourrait jamais comprendre qu'un juge anglais puisse affirmer dans le Ward's case, en 1625, que, pour lui, en tant que juge, Hamburg se trouvait en Angleterre, qu'à condition de connaître les présupposés qui l'ont influencé à faire cette affirmation ou plutôt employer cette fiction.

Maloré la disparition du "jury system", la problématique des conflits de lois garda pendant longtemps la nature juridictionnelle qui avait marqué sa naissance (p. 36 note 4 , p. 37 note 1). Ce ne fut que dans le cas de Robinson v. Bland, tranché en 1760 que Lord Mansfield traca les limites entre les problèmes concernant la compétence juridictionnelle et le choix de la loi applicable à la solution du litige, tandis que les deux autres juges qui connurent de l'affaire estimaient encore que le choix du for anglais entrainait le choix de l'application de la loi anglaise. Il est intéressant de rappeler, par ailleurs, que dans le cas de Foubert v. Turst, tranché en 1703 , les deux parties s'empressèrent d'établir que le litige ne posait absolument pas le problème de l'application de la loi étrangère (p. 39-40). Et il est très symptomatique qu'encore en 1774, dans Mostyn v. Fabricas, le demandeur eût trouvé nécessaire de faire appell à une fiction pour localiser l'acte illicite dont il se plaignait, disant d'abord qu'il s'était passé à Minorque pour ajouter ensuite un "c'est-à-dire" ("videcilet") à Londres (p. 42-44). On y constata que Lord Mansfield écarta la fiction et établit la compétence du for anglais pour connaitre du litige pour des raisons équitables et logiques allant, de la sorte, à l'encontre d'une tradition complètement dépassée par les événements et donc, sans raison de se maintenir. Dans ce sens, se déployèrent les efforts visibles dans plusieurs cas de la phase formative du droit international privé anglais jusqu'au moment où Lord Stovell dans Dalrymple v. Dalrymple, tranché em 1811 (bien qu'il fût dans le sillage de Lord Mansfield dans Holman v. Johnson, tranché en 1775), put affirmer que s'il est vrai que tous les cas soumis aux tribunaux anglais doivent être tranchés en conformité avec la loi anglaise, cela implique l'obéissance aux règles de conflit de lois anglaises dans les litiges concernant les relations privées internationales (p. 46-47). 